ENCYCLOPEDEE Encyclopédie berbère

BERBERE

$27 \mid 2005$

27 | Kairouan - Kifan Bel-Ghomari

\title{
Kef Oum-Touiza
}

C. Roubet et S. Hachi

\section{OpenEdition}

Journals

Édition électronique

URL : http://journals.openedition.org/encyclopedieberbere/1332

DOI : 10.4000/encyclopedieberbere.1332

ISSN : 2262-7197

\section{Éditeur}

Peeters Publishers

\section{Édition imprimée}

Date de publication : 1 août 2005

Pagination : 4143-4146

ISBN : 2-7449-0538-0

ISSN : 1015-7344

Référence électronique

C. Roubet et S. Hachi, « Kef Oum-Touiza », Encyclopédie berbère [En ligne], 27 | 2005, document K38, mis en ligne le 01 juin 2011, consulté le 25 septembre 2020. URL : http://journals.openedition.org/ encyclopedieberbere/1332; DOI : https://doi.org/10.4000/encyclopedieberbere.1332

Ce document a été généré automatiquement le 25 septembre 2020

(c) Tous droits réservés 


\title{
Kef Oum-Touiza
}

\author{
C. Roubet et S. Hachi
}

1 Sur les versants méditerranéens du massif de l'Edough et des monts de la Medjerda, entre Annaba, La Calle et Tabarka-Ouchtata, plusieurs témoins de campements ibéromaurusiens ont été signalés. Aucun d'eux ne conservait de caractéristiques culturelles suffisantes (Gobert et Vaufrey 1932). Aussi, lorsque P. Rodary, Inspecteur des Eaux et Forêts, fit connaître en 1939 la petite station préhistorique de Kef Oum Touiza, entre Lamy et Bordj Ali-Bey, dans le douar Cheffia, là où subsistent des pans effondrés d'une falaise providentielle " Mère de l'entraide " (Feuille $\mathrm{n}^{\circ} 35$ de Munier au $\left.1 / 50000^{\circ}\right)$, celle-ci retint aussitôt l'attention du préhistorien J. Morel, Inspecteur de l'Enseignement primaire, à Bône. Une prospection, des fouilles et des récoltes systématiques faites par P. Rodary et J. Morel livrèrent une documentation ibéromaurusienne substantielle mais déconcertante ainsi que les restes d'un adulte de Mechta-Afalou, (Morel 1953 ; Balout et Briggs 1949).

2 Située à plus de $250 \mathrm{~km}$ à l'est de la nécropole ibéromaurusienne d'Afalou Bou Rhummel* près de Bedjaia, cette station représente pour l'Algérie le repère le plus oriental du type Mechta-Afalou, connu à ce jour. Et, pour la Tunisie, dépourvue de squelettes, une référence culturelle décisive $d u$ frontalier le plus proche, ayant fréquenté le Tell. Tout cela s'est en effet passé bien avant l'entrée des ProtoMéditerranéens au Maghreb, vers le IX millénaire BP et de ceux en particulier venus s'installer dans la grotte néolithique du Kef el-Agab* à une date non encore fixée (Barbin 1951, 1953). Or, il n'existe pas non plus pour ces Proto-Méditerranéens de Tunisie de plus proches ancêtres que ceux issus du peuplement capsien des HautesPlaines du Constantinois.

3 À l'occasion d'une révision documentaire, locale et régionale, s'appuyant sur tous les indices encore disponibles, on essaiera de lever certaines ambiguïtés soulignées par de nombreux auteurs (Balout 1955 ; Vaufrey 1955 ; Camps 1974 ; Hachi 1999 et 2003 (a) et (b)) et l'on tentera de préciser les modalités de circulation et d'occupation de cet espace littoral, tellien et dunaire, par ces deux types humains sapiens, au cours des derniers millénaires. 


\section{Le site}

La station se trouve dans une zone cultivée depuis longtemps (Morel 1953: 175). Limitée à quelques dizaines de mètres carrés entamés par la route et l'oued el-Krenga, elle occupait en 1938 le sommet d'une petite colline gréseuse, couverte d'alluvions sur $1,50 \mathrm{~m}$ d'épaisseur maximum. Ce n'était pas une escargotière, il n'y avait pas beaucoup de cendres. Les restes humains étaient fragmentés et pris dans une gangue terreuse noire, chargée en gravillons. L'individu gisait à $0,80 \mathrm{~m}$ de profondeur, dans ce qui est devenu le fossé de la route. Il était en position accroupie, ou décubitus latéral contracté, les mains croisées sur les jambes fortement repliées, les genoux sur la poitrine. Cette sépulture n'avait reçu aucune offrande, aucun aménagement particulier, il n'y avait pas de traces de rouge funéraire. Les pièces lithiques peu nombreuses apparaissaient éparses en surface, (Rodary 1939 ; Morel 1953 ; Balout et Cabot-Briggs 1949).

\section{La documentation de l'abri du Kef Oum-Touiza}

5 L'homme du Kef Oum-Touiza : Son squelette est celui d'un adulte partiellement conservé, dont la tête seulement a été étudiée après un patient remontage par L. Cabot Briggs. Déposé au CRAPE d'Alger, il porte le premier numéro de l'inventaire général établi par L. Balout (1954). "Les restaurations ont été limitées à l'indispensable pour assurer la solidité » (Balout et Briggs 1949 : 66). C'est à dessein que les restes post-crâniens n'avaient pas été nettoyés de manière à réserver la possibilité d'une étude de la gangue adhérente (note 8, p. 66). L'âge de cet individu masculin ne devait pas dépasser 30 ans, l'usure dentaire était avancée, plusieurs caries étaient observables, les molaires supérieures avaient toutes été perdues ante mortem, et les abcès s'étaient résorbés et cicatrisés. Au maxillaire, une avulsion de l'incisive droite associée à une anomalie de la $2^{\mathrm{e}}$ incisive constitue une particularité de la bouche. À la mandibule sans traces d'avulsion, plusieurs alvéoles sont restées vides, quelques dents subsistent, les branches montantes sont endommagées, les gonions sont extroversés. Sur la face, on observe un front assez haut et incliné, de larges orbites rectangulaires au rebord supérieur marqué, des malaires partiels, assez saillants et massifs, et d'importantes déformations post-mortem qui inclinent la face en arrière et en haut vers la gauche. Trois excellentes planches accompagnent un texte bref. Cet adulte appartient sans conteste au type de MechtaAfalou ayant des caractères atténués (Balout 1954 :100).

\section{Le contexte culturel : son ambiguïté}

6 P. Rodary (1939) puis J. Morel (1953) ont rendu compte de la documentation recueillie que tous deux rattachent à l'Ibéromaurusien. La station ne conservait qu'une seule couche archéologique, sans témoins organiques de faune et de végétaux. Les documents lithiques constituaient donc l'essentiel de la production d'un outillage domestique fait sur place. Les roches exploitées sont locales, issues de formations de l'Eocène voisin: silex, calcédoine, cristal de roche et jaspe ; grès quartzitique et quartz sont présents alentour. Ces différentes matières premières apparaissent sous forme de galets encroûtés dans les alluvions. D'assez bonnes dimensions, aptes au façonnage délicat des 
armatures, conservés sans altérations particulières, ces diverses roches donnent de la diversité à une industrie composite, de constitution macro et microlithique.

7 Le caractère composite de cet ensemble lithique a été bien souligné et attribué par J. Morel au maintien inhabituel de connaissances anciennes dans un contexte ibéromaurusien. "L'industrie du Kef Oum-Touiza... apparaît à l'analyse comme un complexe assez déconcertant où des techniques inégalement évoluées ont été appliquées à des matériaux d'inégale qualité... les formes de tradition levalloiso-atériennes se mêlent aux lamelles et aux microlithes géométriques. La retouche écailleuse moustérienne... voisine avec l'enlèvement abrupt... et la retouche pelliculaire annonciatrice du néolithique» (Morel 1953: 175). Cette opinion n'a pas été soutenue (Balout 1955 ; Vaufrey 1955 ; Camps 1974), l'hypothèse d'un mélange s'est imposée comme dans le cas du gisement du Demnet el-Hassan (La Calle), fouillé et publié par J. Morel (1955).

8 L'inventaire de 1347 pièces réunit les collections de P. Rodary et de J. Morel, il fait apparaître : 63 nucléus, 1006 éclats atypiques, 15 autres éclats, 70 lames et lamelles brutes ; 42 grattoirs, 7 rabots nucléiformes, 3 perçoirs, 4 burins, 11 coches et denticulés, 5 pointes à bord abattu, 26 lamelles à bord abattu et retouches bilatérales, 12 racloirs, 8 pièces géométriques (dont un triangle, une flèche à tranchant transversal, et cinq segments), 1 pointe foliacée biface, 27 éclats retouchés, 1 molette, 44 pierres de jet, et 2 percuteurs (Morel p. 179).

\section{Conclusion}

9 Il y a quelque temps déjà que les origines de l'Homme de Mechta-Afalou se discutent plutôt au Maroc (grotte de Dar-Es-Soltane 2, près de Rabat, et grotte des Contrebandiers, à Témara) (Roche 1976; Debénath, 1976), sur la base de données stratigraphiques, paléontologiques et chronologiques recevables. L'hypothèse d'une ascendance sapiens sapiens, pour l'Homme de culture atérienne de Témara, paraît envisageable, à défaut d'une autre argumentation, puisque nombre de ses traits le rapprochent "des Hommes modernes ou de ceux du type de Mechta-Afalou " (Férembach 1976: 180). Quant à l'Homme de Dar-Es-Soltane 2, au contexte culturel également atérien ${ }^{1}$ (couche 7, Debénath 1976:182, et communication orale du 17-4-2004), il ne diffère des Hommes Mechta-Afalou que par «l'accentuation de certains détails morphologiques ou de dimensions " (Férembach 1979 : 191). On ne s'étonnera pas que cette avancée scientifique surprenne les préhistoriens: dans cette hypothèse phylétique, rien n'annonce l'apparition de la culture si originale de l'Homme Ibéromaurusien. La rupture conceptuelle entre Atérien et Ibéromaurusien est totale.

10 En Algérie, les témoins géologiques et culturels restent encore à multiplier (Betrouni 1983), aucun reste anthropologique lié à l'Atérien n'ayant été découvert. Tout en restant vigilants, on ne peut écarter l'idée - en ce qui concerne l'Homme de MechtaAfalou - d'une continuité anthropologique possible. Pourtant, aujourd'hui encore, il est difficile d'admettre qu'un " homme d'origine autochtone ait été l'auteur d'une culture (et de son industrie) d'origine allochtone" (Hachi 2003(a) : 20-21), inaugurant au Maghreb une expression artistique exceptionnelle et inattendue (Hachi 2003(b) ; Roubet in Hachi, 2003(b)). L'ancienneté des travaux en Algérie et au Kef Oum-Touiza, en particulier, oblige à réévaluer tous les thèmes documentaires. Le sujet reste ouvert. 


\section{BIBLIOGRAPHIE}

BALOUT L. et L. CABOT-BRIGGS 1949. «Tête osseuse du Kef Oum-Touiza ». Trav. du Lab. d'Archéol. et d'Anthrop. Préhistoriques du Musée du Bardo d'Alger, I, 7 p., III pl. h.t.

BALOUT L. et L. CABOT-BRIGGS 1949. « Tête osseuse du Kef Oum-Touiza ». Bull, de la Soc. d'Hist. nat. de l'Afrique du Nord, t. XL, pp. 64-70, III pl. h.t.

BALOUT L., 1954. « Les Hommes préhistoriques du Maghreb et du Sahara. Inventaire descriptif et critique, Paléolithique, Épipaléolithique et Néolithique ». Libyca, t. 2 (p. 100).

BALOUT L., 1955. Préhistoire de l'Afrique du Nord. Essai de chronologie. Paris, AMG.

BARDIN P., 1951. « Note sur le gisement néolithique de la grotte du Kef el-Agab ». Bull, de la Soc. des Sc. Nat. de Tunisie, t. 4, pp. XXXV et 23-24.

BARDIN P., 1953. « Gisement néolithique de la grotte du Kef el-Agab, Tunisie ». Libyca, t.

1,pp. 271-308.

BÉTROUNI M., 1983. Le Pléistocène supérieur du littoral ouest algérois. Thèse de doctorat de $3^{\mathrm{e}}$ cycle, Faculté des Sciences de Luminy, Aix-Marseille II, 202 p., XIII pl. 1 carte h.t.

CAMPS G, 1974. Les civilisations préhistoriques de l'Afrique du Nord et du Sahara. Paris, Doin.

DEBÉNATH A., 1976. « Le site de Dar-Es-Soltane 2, à Rabat (Maroc) ». Bull, et Mém. de la Soc. d'Anthrop. de Paris, t. 3, série XIII, pp. 181-182.

FÉREMBACH D., 1976. « Les restes humains de la grotte de Dar-Es-Soltan 2, (Maroc), campagne 1975 ». Bull, et Mém. de la Soc. d'Anthrop. de Paris, t. 3, série XIII, pp. 183-193.

FÉREMBACH D., 1976. «Les restes humains atériens de Témara. (Campagne de 1975) ». Bull, et Mém. de la Soc. d'Anthrop. de Paris, t. 3, série XIII, pp. 175-180.

GOBERT E.-G. et VAUfREY R., 1932. « Deux gisement extrêmes d'Ibéromaurusien ». L’Anthrop., t. XLII, pp. 449-490.

HACHI S., 1999. L'Homme de Mechta-Afalou, Cro-Magnon de l'Afrique du Nord durant les 20 derniers millénaires : évolution culturelle et devenir. Thèse d'Habilitation à Diriger des Recherches, Université P. Valéry III, Montpellier, 4 volumes.

HACHI S., 2003a. Les cultures de l'Homme de Mechta-Afalou. Le gisement d'Afalou Bou-Rhummel (massif des Babors, Algérie). Les niveaux supérieurs 13 000-11 000 BP., CNRPAH.

HACHI S., 2003b. Aux origines des Arts premiers en Algérie. Les figurines et les objets modelés en terre cuite de l'Abri-sous-roche préhistorique d'Afalou, Babors, Algérie, 18 000-11 000 BP CNRPAH, Alger, n.s., 6.

MARCHAND H.F., 1951. « Les documents paléo-anthropologiques de la Cheffia », Mélanges d'Anthropologie et de Sociologie nord-africaine, Alger, pp. 71-89.

MOREL J., 1953. « L'outillage lithique de la station du Kef Oum-Touiza dans l'Est Constantinois ». Libyca, t. 1, pp. 157-179, VI pl.

MOREL J., 1952 (1955). « La station préhistorique du Demnet el-Hassan, dans la commune mixte de La Calle (département de Constantine), et le problème de l'Ibéro-Maurusien ». Actes du II ${ }^{e}$ Congrès panaf. de Préhistoire d'Alger, pp. 631-639. 
ROCHE J., 1976, «Chronostratigraphie des restes atériens de la grotte des Contrebandiers à Témara (Province de Rabat) ». Bull, et Mém. de la Soc. d'Anthrop. de Paris, t. 3, série XIII, pp. 165-173.

RODARY P., 1939. «Contribution à la recherche des stations préhistoriques de la région de Bône et de La Calle ». Congrès des Sociétés Savantes, Tunis.

ROUBET C, 2003 b. « Avant-Propos ", in Hachi S., Aux origines des Arts premiers en Algérie. Les figurines et les objets modelés en terre cuite de l'Abri-sous-roche préhistorique d'Afalou, Babors, Algérie, 18000-11000 BP. CNRPAH, Alger, n.s., 6, pp. 10-12.

VALLoIs Dr H.-V, 1953. « Note sur les ossements humains du Kef el-Agab » in Bardin P., « Gisement néolithique de la grotte du Kef el-Agab, Tunisie ». Libyca, t. 1, pp. 271-308.

VAUfReY R., 1955. Préhistoire de l'Afrique. T. 1 : Le Maghreb. Paris, Masson, Publ. de l'Instit. des Hautes Études de Tunis, vol. 4.

\section{NOTES}

1. Nous remercions notre collègue et ami André Debénath pour cette information inédite, tant attendue, fournie le 17/0/2004.

INDEX

Mots-clés : Ibéromaurusien, Préhistoire 